\title{
Fundamentos para la gestión del conocimiento en grupos de investigación.
}

\section{Foundations for the Knowledge Management in the Investigation Groups.}

\author{
Carlos Humberto Carreño Díaz ${ }^{2}$, Sonia Cristina Gamboa Sarmiento ${ }^{3}$ \\ ${ }^{1}$ Universidad Industrial de Santander, Colombia.
}

Artículo recibido en el mes de Febrero de 2014; artículo aceptado en el mes de Junio de 2014

Citación del artículo: Carreño, C. H. \& Gamboa, S. C. (2014). Fundamentos para la gestión del conocimiento en grupos de investigación. I+D Revista de Investigaciones, 3(1), 40-50.

\section{Resumen}

En el presente documento se realiza un análisis a los grupos de investigación tanto como unidades de un sistema nacional e internacional de ciencia y tecnología, como organizaciones que llevan a cabo actividades de administración y gestión de recursos, que requieren del reconocimiento y continuo mejoramiento de los procesos que soportan las actividades de investigación. Para la mejora en la calidad de su dinámica investigativa, es indispensable el reconocimiento de los elementos facilitadores de la gestión del conocimiento para el desarrollo de ventajas competitivas que favorezcan la generación de resultados de investigación tangibles y verificables, los cuales respondan a problemas contextuales de su disciplina y que puedan ser transformados en productos tecnológicos, fruto de un proceso científico. En resumen, se busca presentar los elementos que soportan la dinámica de investigación de los grupos y en los cuales se fundamentan los procesos de gestión del conocimiento que se derivan de su actividad investigativa.

Palabras clave: Gestión del conocimiento, memoria organizacional, grupos de investigación.

\footnotetext{
${ }^{1}$ Artículo de reflexión, de enfoque cualitativo, resultado de un proyecto de investigación, desarrollado en grupo de Investigación denominado Filosofía y Enseñanza de la Filosofía, Universidad Industrial de Santander (UIS) (Colombia). Dirección: Carrera 27 Calle 9, PBX: (7)6344000, Bucaramanga.

${ }^{2}$ Ingeniero de Sistemas, Universitaria de Investigación y Desarrollo (UDI) (Colombia). Magíster en Ingeniería de Sistemas e Informática, Universidad Industrial de Santander (UIS). Docente-Investigador del grupo Filosofía y Enseñanza de la Filosofía, Universidad Industrial de Santander (UIS) (Colombia). Correoelectrónico: carlosh.carreno1@correo.uis.edu.co ${ }^{3}$ Ingeniera de Sistemas, Universidad Industrial de Santander (UIS) (Colombia). Doctor en Educación, Universidad Pedagógica Nacional (UPN). Docente-investigador del grupo Filosofía y Enseñanza de la Filosofía, Universidad Industrial de Santander (UIS) (Colombia).Correo electrónico: scgamboa@uis.edu.co
} 


\section{Abstract}

In this study the investigation groups are analyzed as well a national and international Science and Technology unit, as an organizations which administer resources and require institutional recognition and continuous investigation standards improvement. In the making of the investigation process better, it is essential the recognizing of the knowledge management facilitator elements in the developing of competitive advantages in the production of concrete and verifiable researching results in its own areas of knowledge responding to the contextual problems and transforming those outcomes in technological products of the scientific process. In summary, the knowledge management components of the dynamics of an investigation group are presented.

Keywords: Knowledge management, organizational memory, investigation groups.

\section{Introducción}

En las sociedades contemporáneas, el desarrollo de los Estados depende de manera directa de sus procesos de investigación, desarrollo e innovación, por lo cual, el saber se ha convertido en la principal fuerza de producción (Lyotard, 1979). La investigación científica se instaura como una actividad crítica que determina el desarrollo de los países de las sociedades actuales y que depende de la competencia de sus investigadores y de la aplicación que se le dé al conocimiento generado por éstos. De manera general, las actuales políticas estatales apuntan hacia la creación de
Sistemas nacionales de innovación e investigación, los cuales, de acuerdo con Rip y Van der Meulen (1996) "están constituidos por los ejecutores de la investigación (individuos, grupos, instituciones), otras organizaciones e instituciones, interacciones, procesos y procedimientos" (p. 18), por lo que, es prioritario desarrollar modelos que permitan caracterizar los actores involucrados en el proceso de investigación científica, e identificar los factores que afectan la productividad de tales sistemas. Los grupos de investigación se identifican como elementos clave, cuyos procesos investigativos principales deben ser analizados. La actividad investigativa en dichos grupos posee características particulares, principalmente por la renovación constante, no sólo de sus miembros, sino también del mismo entorno en el cual el grupo se desenvuelve. La gestión adecuada del conocimiento en el grupo - tanto interno como externo - favorece la creación de productos de conocimiento y sirve como estrategia de la calidad de los proyectos de investigación en un entorno cada vez más exigente. A partir de esto, surge la siguiente pregunta: ¿Cuáles son las principales actividades que se llevan a cabo en los grupos de investigación para la gestión del conocimiento y cómo se relacionan en ellas los diferentes actores involucrados en los procesos de investigación?.

Para dar respuesta a esto, es importante analizar en primera instancia los elementos que fundamentan la gestión del conocimiento en su actividad investigativa. Para ello, se iniciará con la revisión de las actividades de gestión del conocimiento en el contexto de grupos de investigación, a partir del estudio de su estructura y dinámica. 


\section{Dinámica de la actividad investigativa}

De acuerdo con Colciencias (2012), un grupo de investigación corresponde al "conjunto de personas que se reúnen para realizar investigación en una temática dada, formulan uno o varios problemas de su interés, trazan un plan estratégico de largo o mediano plazo para trabajar en él y producir unos resultados de conocimiento sobre el tema en cuestión" (p. 16). El desarrollo de los proyectos de investigación en un grupo, genera una confluencia de experiencias individuales, debido al interés que tienen en común los investigadores acerca de ciertas problemáticas, dando lugar a un "espacio estructurado de problemas $u$ objetos de investigación relevantes dentro de un campo de conocimiento que favorece la creación de grupos de investigación"(Colciencias, 2006).

En primera instancia, se debe examinar la forma como los grupos de investigación se encuentran organizados en torno a sus proyectos, a partir de los cuales se generan productos que no sólo ofrecen aportes significativos al estado del arte de un área de conocimiento, sino que también están asociados al desarrollo económico, las transformaciones sociales mediante apropiación y uso de dichos productos de conocimiento por parte de la sociedad. Gracias a la diversidad de proyectos, es posible propiciar escuelas de formación de investigadores cuyos propósitos, a pesar de ser diversos, convergen en resultados comunes, compartiendo recursos organizacionales y siguiendo un conjunto de políticas o normas establecidas por el grupo.

De manera general, los grupos están conformados principalmente por equipos de investigadores, comprendidos tanto por profesionales con formación avanzada maestría y doctorado - como por estudiantes de programas de pregrado y postgrado, quienes avanzan en un proceso de formación como investigadores y quienes optan por la investigación como ejercicio profesional. En su actividad investigativa, uno o varios investigadores tienen a su cargo un determinado número de colaboradores y aprendices que, bajo su guía, se forman como investigadores, a su vez que fortalecen competencias para el ejercicio profesional y académico. Estos actores confluyen en programas, líneas y proyectos de investigación, los cuales buscan validar o transformar conocimientos, ya sea para el establecimiento de teorías, como para desarrollar o refinar dispositivos tecnológicos que respondan a problemas contextuales, contribuyendo así al cuerpo de conocimiento de diversas disciplinas.

Gamboa Sarmiento, Otero Riaño y Torres Camacho (2012), representan la forma como se relacionan en el contexto universitario los actores y sus actividades de investigación, así como los medios de publicación y exteriorización de resultados. Como se puede ver en la figura 1, se expone la relación entre las funciones de investigación, docencia y extensión propias de la misión universitaria. En primer lugar se encuentra el componente formativo de la investigación, compuesto principalmente por estudiantes en formación en diferentes niveles académicos, articulados en los semilleros de investigación del grupo; por otra parte, los investigadores expertos soportan las líneas de investigación con el apoyo de proyectos 
doctorales realizados por investigadores en formación doctoral. La exteriorización de los resultados de investigación se realiza en los diferentes niveles estructurales mediante la publicación en medios de comunicación científica, así como a través de la participación en seminarios, eventos o actividades de extensión.

Figura 1. Desarrollo de la investigación en el contexto Universitario. Concebida por el grupo de investigación Filosofía y Enseñanza de la Filosofía.

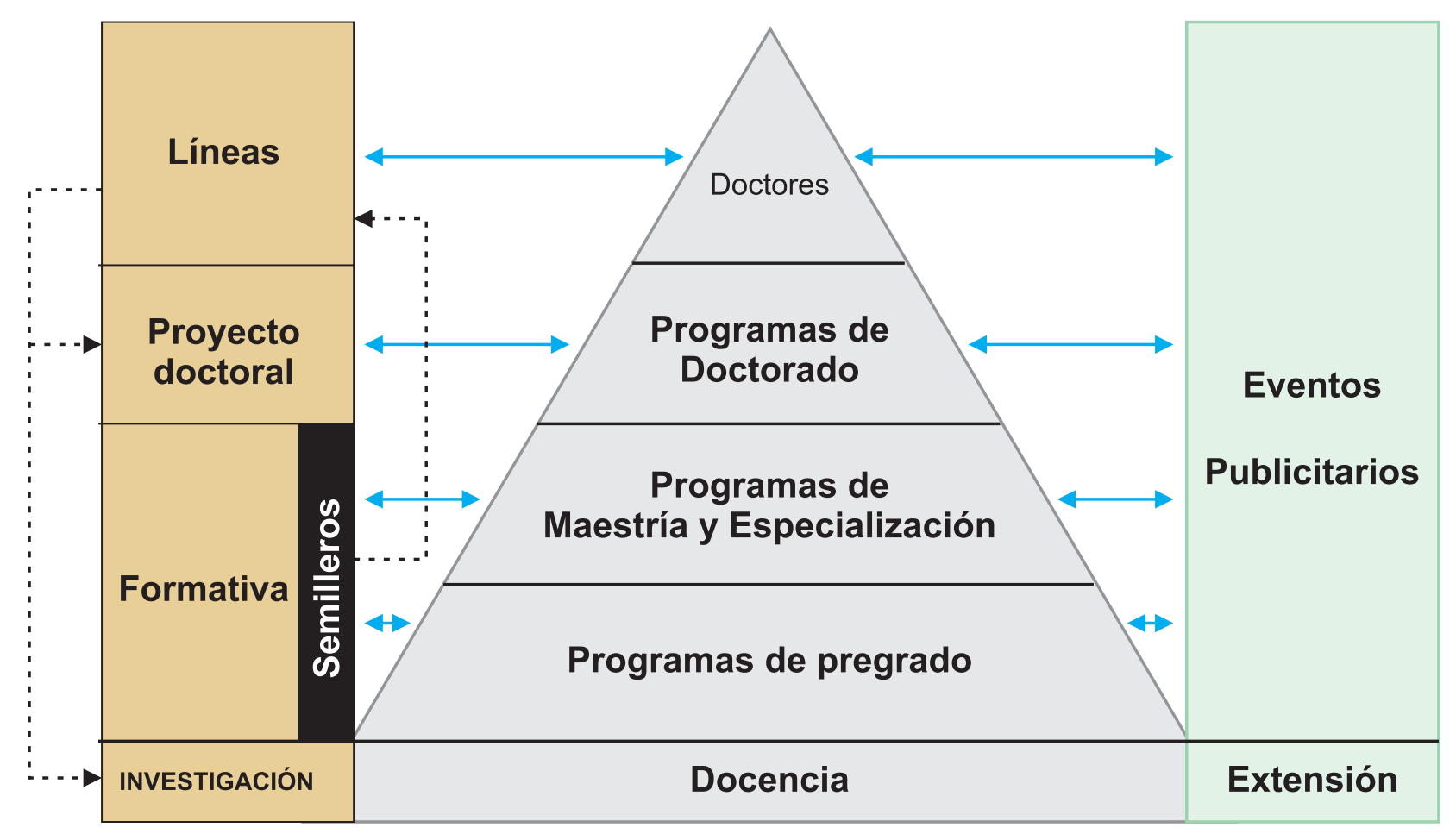

Fuente: Gamboa Sarmiento, Otero Riaño y Torres Camacho (2012, p. 37)

Además de los aspectos involucrados en las actividades propias de los grupos de investigación, existen elementos relacionados a su estructura, organización y sostenibilidad. Londoño (2005), identifica los elementos de conformación de los grupos, relacionándolos a través de los espacios de conversación e interacción en los que participan sus miembros y el modo como éstos se relacionan; por otra parte, identifica elementos de pervivencia, los cuales definen el ser y estar del grupo de investigación en el contexto académico y social (ver tabla 1). De tal forma, los grupos de investigación deberán establecer medios que les permitan garantizar que estos elementos se incorporen en su cultura organizacional y formen parte de sus procesos investigativos, favoreciendo la calidad de sus proyectos realizados. 
Tabla 1. Conformación y pervivencia en grupos de investigación.

\section{CONFORMACIÓN}

(espacios de conversación e interacción)

\section{PERVIVENCIA}

(Ser y estar en el mundo)
Organización: esquemas organizativos; asignación de tareas; cultura organizacional; diversidad proyectos y perspectivas.

Estructura: Investigadores expertos y en formación asociados a proyectos, programas y líneas de investigación.

Participación: intercambio de impresiones particulares de manera formal e informal: seminarios, talleres, discusiones, entre otros.

Espacios: lugares físicos o virtuales abiertos, flexibles e integrados para la comunicación, conversación y discusión.
Liderazgo: el director de grupo y la gestión de recursos y directrices; directores de proyectos y la formación de investigadores.

Mecanismos de renovación: alternancia constante de miembros; pasantes; reestructuración de líneas y programas.

Plan estratégico: misión y visión organizacional, definición de líneas de investigación, portafolio de proyectos.

Sentido académico y social: aportes al área de conocimiento; apropiación social del conocimiento; impulso de transformaciones sociales; innovación.

Sostenimiento económico: gestión de recursos económicos; financiación; convenios; integración con el sector productivo.

Fuente: Adaptado de Londoño (2005)

\section{La gestión del conocimiento en los grupos de investigación}

De acuerdo con Wiig (1997), la gestión del conocimiento incluye "comprender, enfocar, y administrar de manera sistemática, explícita y deliberada la construcción, renovación y aplicación del conocimiento - es decir, gestionar los procesos efectivos del conocimiento" (p. 2). Alavi y Leidner (2001), agregan que la gestión del conocimiento involucra "identificar $y$ aprovechar el conocimiento colectivo en una organización, para ayudar a la organización a competir" (p. 113). Para cumplir esto, es indispensable contar con las personas, procesos y tecnologías adecuadas que permitan optimizar de forma dinámica el nivel de competencia y aprendizaje de los miembros de la organización, mediante el desarrollo de una inteligencia colectiva (Maier, 2007). 
En ese sentido, la gestión del conocimiento es de gran interés no sólo para las ciencias de la información, sino también para áreas relacionadas con administración, la ingeniería industrial, entre otras. El soporte ofrecido por las ciencias de la información y la ingeniería de sistemas, por ejemplo, ha favorecido la implementación de herramientas computacionales que se adapten a los entornos organizacionales, suscitando el desarrollo y ejecución de diferentes enfoques y perspectivas en gestión del conocimiento. En el desarrollo de este proyecto, se estudió la dinámica de la gestión del conocimiento en los grupos de investigación, con lo que se pudo establecer un modelo inicial de la relación de las actividades principales en las cuales los grupos centran sus procesos investigativos (ver figura 2).

Figura 2. Procesos de gestión del conocimiento en grupos de investigación

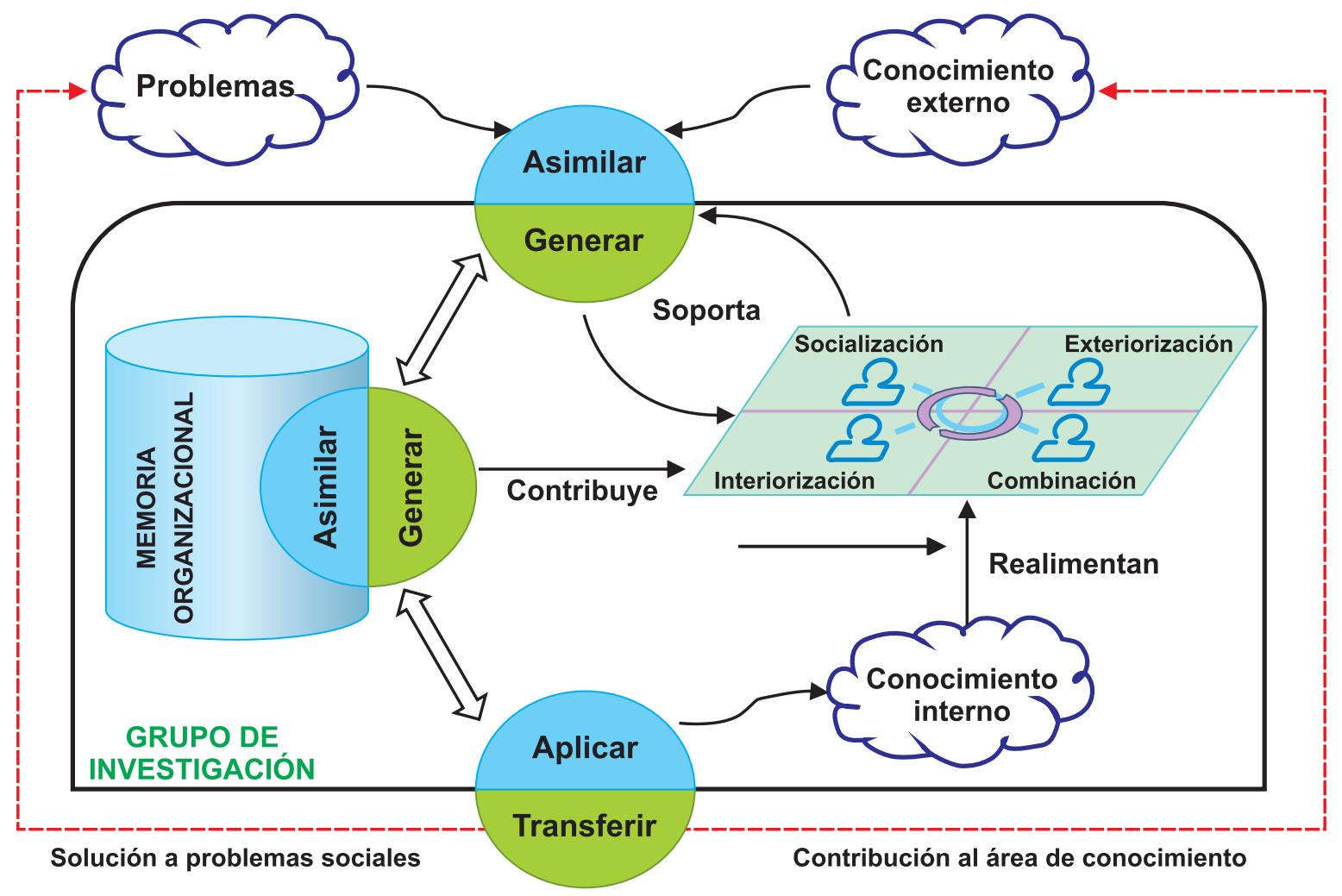

Fuente: Autor

En el contexto de los grupos de investigación, la gestión del conocimiento parte de la identificación de problemas - de origen interno o externo, que responden a necesidades sociales o académicas - que son analizados y asimilados por el grupo de investigación para el inicio del proceso de generación de conocimiento, el cual puede estar soportado tanto por las interacciones de individuos, como por la utilización de herramientas computacionales. El conocimiento generado en el grupo, alimenta la memoria organizacional, la cual puede ser almacenada en repositorios de conocimiento - explícito - y volviéndose parte de la memoria colectiva tácita - ya no sólo del individuo, sino del mismo grupo. La diseminación del conocimiento en la 
organización, contribuye tanto en los procesos de generación de conocimiento, como en la aplicación de dicho conocimiento en proyectos de investigación.

Por otra parte, la aplicación del conocimiento generado, suscita también conocimiento interno, tanto tácito como explícito, el cual debe ser capitalizado e incluido nuevamente en los procesos de investigación en busca del complimiento de las metas organizacionales (Jaime, Gardoni, \& Mosca, 2006). Asimismo, el proceso de aplicación genera resultados que se materializan como productos de conocimiento, los cuales se transfieren a las comunidades involucradas e interesadas, generando nuevas necesidades o problemáticas a estudiar. De ser aceptados como válidos por parte de la comunidad científica, estos resultados propician la generación de conocimiento externo, el cual debe ser identificado e interiorizado, siendo así, asimilado de vuelta en el grupo de investigación.

\section{Conclusiones y trabajo futuro}

Para gestionar el conocimiento en grupos de investigación, se debe considerar que las formas de trabajo, los tipos de investigaciones y sus objetivos, difieren de un grupo de investigación a otro, principalmente por su autonomía y flexibilidad de su organización, por lo que la definición de la estructura organizativa del grupo requiere principal atención. La estructura organizacional es un aspecto moderador de la gestión del conocimiento, ya que permite definir las relaciones formales, la asignación de actividades y el uso de recursos entre los miembros de la organización, requiriendo de reglas definidas formalmente, políticas y procedimientos estandarizados (Allameh, Zare, \& Davoodi, 2011).

En ese sentido, debido a la dinámica de las diferentes áreas del saber, no existe una única forma "adecuada" de organización en los grupos de investigación. Esto se debe a que diferentes grupos poseen formas diversas de organizarse para abordar los problemas concretos que desean solucionar. Se observa, sin embargo, que existen roles comunes que se encuentran en cada grupo de investigación, como un director de grupo, los investigadores que soportan las líneas de investigación y orientan el trabajo de investigadores asociados, e investigadores en formación. Puesto que la dinámica de participación e interacción existente en cada grupo, son moderadas por la flexibilidad o rigidez de las estructuras organizacionales definidas (Mejía Correa, 2007), cada grupo de organización debe definir los niveles estructurales y canales de comunicación que faciliten los procesos de gestión del conocimiento.

Se deberán tener en cuenta tanto los mecanismos de gestión de estas estructuras organizativas, como la identificación y aprovechamiento del capital intelectual en cada una de sus formas: el capital humano investigadores, estudiantes y personal auxiliar -, el capital estructural - recursos bibliográficos y espacios físicos o virtuales de interacción - y el capital relacional - relaciones con la comunidad científica como convenios con otros grupos o empresas - (Rodríguez Castellanos, Araújo de la Mata, \& Urrutia Gutiérrez, 2001). La gestión del capital intelectual, representado en el 
conocimiento científico-técnico - recurso fundamental y producto de las actividades de los grupos de investigación -, permite el alcance de nuevas teorías, técnicas, proyectos de investigación, desarrollo e innovación, además de favorecer el proceso de aprendizaje organizacional (Yang et al. 2006, 2009).

Por ello, es importante el establecimiento de políticas para valorar, proteger, difundir y comercializar el conocimiento generado en el grupo, como los resultados mismos de la investigación, de manera que se promueva la apropiación tanto interna como social de dichos resultados por los miembros del grupo, buscando que éste sea exteriorizado a través empresas e instituciones mediante proyectos de extensión e innovación. La conformación de la cultura organizacional es un factor importante en la gestión eficaz del conocimiento, ya que proporciona un entorno adecuado para el intercambio de conocimientos y el apoyo a las actividades desarrolladas por el grupo (Allameh et al., 2011). Debido al cambio constante generado por los avances en las diferentes áreas del conocimiento, la cultura organizacional en un grupo de investigación debe estar orientada al cambio, a la colaboración interdisciplinar, la vigilancia tecnológica, así como a la difusión y aprovechamiento del conocimiento existente de los miembros más antiguos del grupo, para evitar reinvención de soluciones o caer en problemas que ya han sido solucionados anteriormente.
El grado de liderazgo que existe en la organización, también juega un papel fundamental. En los grupos de investigación, el liderazgo es ejercido principalmente por los investigadores adscritos a los diferentes proyectos y por su director, quienes soportan las actividades científicas y organizan, gestionan y guían el trabajo realizado por los investigadores en formación. Los líderes de grupo propician la adquisición de recursos y el uso estratégico de los mismos en el grupo de investigación, estimulan el trabajo realizado por los miembros del grupo, coordinan el proceso de investigación y realizan actividades externas, para relacionar al grupo de investigación con otras entidades como la empresa privada $u$ organizaciones que soportan o realizan investigación - buscando garantizar la legitimidad científica de sus proyectos, y mejorar la reputación y la visibilidad del grupo.

Como trabajo futuro se buscará establecer un modelo que describa cómo los elementos anteriormente mencionados $-\mathrm{y}$ relacionados en la figura 2 - interactúan con los procesos de gestión del conocimiento en los proyectos investigativos, y cómo a través de la utilización de TIC, se pueden definir entornos virtuales, mediante los cuales investigadores expertos y en formación puedan participar en la conformación de una memoria colectiva, por medio de herramientas para la comunicación, coordinación y colaboración. 
Figura 2. Componentes a considerar en la investigación y gestión del conocimiento

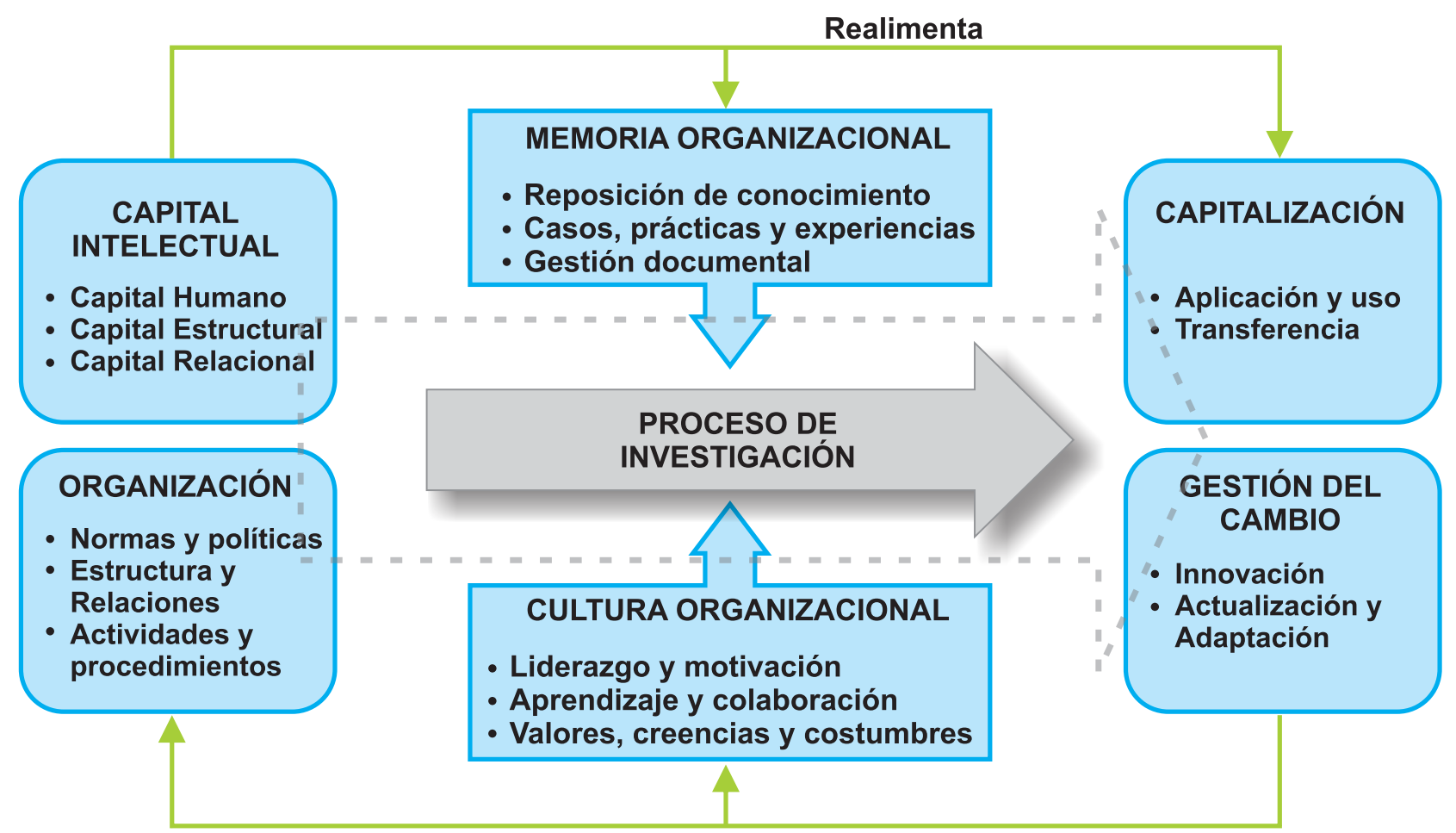

Fuente: Autor

\section{Referencias}

Alavi, M., \& Leidner, D. E. (2001). Review: Knowledge management and knowledge manage systems: conceptual foundations and research issues. MIS Quarterly, 25(1), 107-136.

Allameh, S. M., Zare, S. M., \& Davoodi, S. M. R. (2011). Examining the impact of KM enablers on knowledge management processes. Procedia Computer Science, 3, 1211-1223. doi:10.1016/j.procs.2010.12.196

Colciencias índice para la medición de Grupos de Investigación Tecnológica o de Innovación (2006).
Colciencias Modelo de medición de Grupos de Investigación Tecnológica o de Innovación Año 2008 (2012).

Gamboa Sarmiento, S. C., Otero Riaño, N. R., \& Torres Camacho, J. I. (2012). Aplicación web para modelo de gestión del conocimiento propuesto por el grupo de investigación filosofía y enseñanza de la filosofía. Universidad Industrial de Santander.

Jaime, A., Gardoni, M., \& Mosca, J. (2006). From quality management to knowledge management in research organizations. International Journal of innovation management, 10(2), 197-215. 